\title{
Réponse agro-morphologique de 14 génotypes de sorgho grains sucrés du Burkina Faso à la fertilisation minérale.
}

TIENDREBEOGO Josiane*, SAWADOGO Nerbéwendé1, KIENDREBEOGO Timbilfou², KIEBRE Zakaria1, SAWADOGO Boureima1', KIEBRE Mariam¹, ZERBO Afoussatou'1, NANEMA Kiswendsida. Romaric $^{1}$ et SAWADOGO Mahamadou'.

1 Université Joseph KI-ZERBO, Ecole Doctorale Sciences et Technologie, Laboratoire Biosciences, Equipe Génétique et Amélioration des Plantes, 03 BP 7021, Ouagadougou 03, Burkina Faso.

2 Institut de l'Environnement et de la Recherche Agricole (INERA), Station de Recherche de Farako-Bâ, BP 910 Bobo Dioulasso, Burkina Faso.

*Auteur correspondant Email : josi tiendre@yahoo.fr , Tel : (00226) 72705686/ 0022666961030

Original submitted in on $5^{\text {th }}$ November 2019. Published online at www.m.elewa.org/journals/ on $31^{\text {st }}$ January 2020 https://doi.org/10.35759/JABs.v145.3

\section{RESUME}

Le sorgho grains sucrés est une culture produite essentiellement pour ses grains sucrés et riches en oligoéléments consommés au stade grains pâteux.

Objectif: L'étude vise à déterminer l'effet de la fertilisation minérale (NPK) sur l'expression des paramètres agro-morphologiques du sorgho grains sucrés [Sorghum bicolor (L.) Moench].

Méthodologie et résultats: L'essai a été réalisé en saison pluvieuse selon un dispositif en split plot avec trois répétitions. Quatorze génotypes de sorgho grains sucrés ont été soumis à trois niveaux de fertilisation à savoir $100 \mathrm{~kg} \mathrm{NPK/ha} \mathrm{(TR);} 125 \mathrm{~kg} \mathrm{NPK/ha} \mathrm{(T1)} \mathrm{et} 150 \mathrm{~kg} \mathrm{NPK/ha} \mathrm{(T2).} \mathrm{Au} \mathrm{total,} \mathrm{neuf} \mathrm{variables}$ quantitatives relatives à la phénologie de la plante, au tallage et aux rendements grains et paille ont été mesurées. Les résultats ont révélé que seule la teneur en matière sèche de la paille (TMS) a présenté une différence significative $(p<0.05)$ en fonction du facteur traitement. La dose TR (100 kg NPK/ha) a été optimale pour la production en grains et en paille du sorgho grains sucrés. Les rendements à l'hectare pour l'ensemble des trois traitements ont varié respectivement de 3397,092 kg (MTC2) à 8969,937 kg (SBR7) pour le grain et de $4154,948 \mathrm{~kg}$ (MTC2) à 12683,594 kg (SBR7) pour la paille.

Conclusion et applications : II ressort de cette étude que le niveau de fertilisation a eu très peu d'influence sur la plupart des paramètres agro-morphologiques de la plante. La dose de $100 \mathrm{~kg} \mathrm{NPK/ha} \mathrm{est} \mathrm{une} \mathrm{dose}$ optimale pour la production de cette culture. Cela permet d'orienter le producteur en matière de fertilisation et sur le choix des génotypes de sorgho grains sucrés à double usage (grains et fourrage).

Mots clés: Sorgho grains sucrés, génotypes, NPK, diversité, Burkina Faso. 


\section{Agromorphological response of 14 genotypes of sweet grain sorghum from Burkina Faso to mineral fertilization \\ ABSTRACT}

Sweet grain sorghum is a crop produced mainly for its sweet grains and rich in trace elements consumed in the pasty grains stage.

Objective: The study aims to determine the effect of mineral fertilization (NPK) on the expression of agromorphological parameters of sorghum sweet grains [Sorghum bicolor (L.) Moench].

Methodology and results: The experiment was carried out in the rainy season according to a split plot design with three repetitions. Fourteen sweet grain sorghum genotypes were submitted to three levels of fertilization, defined as $100 \mathrm{~kg} \mathrm{NPK} / \mathrm{ha}$ (TR); $125 \mathrm{~kg} \mathrm{NPK} / \mathrm{ha}$ (T1) and $150 \mathrm{~kg} \mathrm{NPK/ha} \mathrm{(T2).} \mathrm{A} \mathrm{total} \mathrm{of} \mathrm{nine}$ quantitative variables related to plant phenology, tillering and grain and straw yields were measured. The results revealed that only the dry matter content of straw (TMS) showed a significant difference $(p<0.05)$ depending on the treatment factor. The TR dose (100 kg NPK/ha) was optimal for grain and straw production of sweet grain sorghum. Yields per hectare for all treatments varied from $3397.092 \mathrm{~kg}$ (MTC2) to $8969.937 \mathrm{~kg}$ (SBR7) and from $4154.948 \mathrm{~kg}$ (MTC2) to $12683.594 \mathrm{~kg}$ (SBR7) respectively for grain and straw. This study shows that the level of fertilization has had very little influence on most of the agromorphological parameters of the plant. The $100 \mathrm{~kg}$ NPK/ha dose is an optimal dose for the production of this crop. This allows to guide the producer in fertilization and the choice of sweet grain sorghum genotypes for dual-use (grains and feed).

Key words: Sweet grain sorghum, genotypes, NPK, diversity, Burkina Faso.

\section{INTRODUCTION}

Dans les pays sahéliens, notamment le Niger, le Mali, le Sénégal et le Burkina Faso, les céréales constituent la base de l'alimentation des populations (FAO, 2017). Parmi ces céréales, le sorgho, classé 3ème en terme de production grains, constitue l'aliment de base de millions de personnes (Gueye et al., 2016) et un excellent fourrage pour les animaux. Cependant, sa culture fait face à d'énormes contraintes inhérentes à l'agriculture des zones Soudano-Sahéliennes d'Afrique, à savoir les variabilités inter et intra-annuelles de la pluviométrie (Hansen, 2002), accentuées par les effets du changement climatique (Slingo et al., 2005) et la faible fertilité des sols (Vanlauwe et al., 2010). Du fait de la forte pression démographique, les jachères de longue durée sont moins pratiquées (Zeinabou et al., 2014). Ainsi, la culture continue couplée à l'exportation des nutriments par des cultures exigeantes (coton et maiis) sur des sols pauvres, sont des facteurs qui maximisent les risques de baisse de la fertilité des sols, avec comme corollaires la baisse des rendements agricoles et plus globalement la fragilisation de la sécurité alimentaire (Segda et al., 2001; Coulibaly et al., 2012; IIFA, 2014). Au Burkina Faso, ces contraintes se traduisent par une baisse du taux de la matière organique (< $1 \%$ pour $60 \%$ des sols), une désaturation du complexe absorbant et une acidification des sols (Koulibaly, 2014). Au Burkina Faso, le sorgho grains sucrés est en effet cultivé pour ses grains pâteux sucrés riches en fructose consommés dans l'alimentation humaine (Sawadogo et al., 2018), son fourrage utilisé dans l'alimentation du bétail. La plupart des études antérieures menées sur le sorgho grains sucrés du Burkina Faso se sont surtout intéressées à sa variabilité agro-morphologique (Nebié et al., 2012, Sawadogo et al., 2014a, Sawadogo et al., 2014b, Tiendrébéogo et al., 2018), à sa diversité génétique à l'aide des marqueurs moléculaires (Sawadogo et al., 2017) et à la composition glucidique des grains (Sawadogo et al., 2017). Cependant, aucune étude n'avait abordé la réponse du sorgho grains sucrés à la fertilisation. Pourtant, les apports en nutriments pourraient constituer une alternative à l'appauvrissement des sols pour augmenter la productivité des cultures et répondre à une demande alimentaire sans cesse croissante. La présente étude vise donc (i) à déterminer l'effet des fertilisants sur les paramètres morphologiques et les performances agronomiques du sorgho et (ii) à identifier la dose optimale de fertilisant pour maximiser les corrélativement les rendements grains et paille. 


\section{MATERIEL ET METHODES}

Matériel végétal: Quatorze génotypes de sorghos grains sucrés du germoplasme du laboratoire Biosciences de l'Université Joseph KI-ZERBO ont servi à l'étude. Ils ont été sélectionnés en raison de leurs bonnes performances agronomiques à savoir le diamètre moyen des tiges $(2,709 \mathrm{~cm})$, poids moyen des panicules $(161,056 \mathrm{~g})$ et poids grains moyen des panicules $(134,036 \mathrm{~g})$ élevés et nombre de talles utiles moyen par pieds important $(2,348)$ (Tiendrébéogo, 2018).

Site expérimental : L'expérimentation a été menée en saison pluvieuse, lors de la campagne agricole 20152016 dans la station expérimentale de l'Institut du Développement Rural située à Gampèla de coordonnées géographiques $12^{\circ} 1^{\prime}$ de longitude Ouest et $12^{\circ} 24^{\prime}$ de latitude Nord. Les sols du site ont une texture à dominance limono-sableux et de faible fertilité physico-chimique (Kiébré, 2016). En effet, l'analyse du sol réalisée par le BUNASOL (Bureau National des sols) montre que le $\mathrm{pH}$ de l'eau du sol est acide $(5,26$ $5,64)$, les taux d'azote $(0,046-0,071 \%)$, de phosphore assimilable $(9,71-10,91 \mathrm{ppm})$ et de matière organique (0,957-1,646\%) faibles. Le climat est de type Nord soudanien. En 2016, la température moyenne mensuelle a oscillée entre $25^{\circ} \mathrm{C}$ et $33,3^{\circ} \mathrm{C}$ et la station a bénéficié de $335,3 \mathrm{~mm}$ de pluies. La figure1 présente l'évolution de la pluviométrie au cours de l'année d'étude.

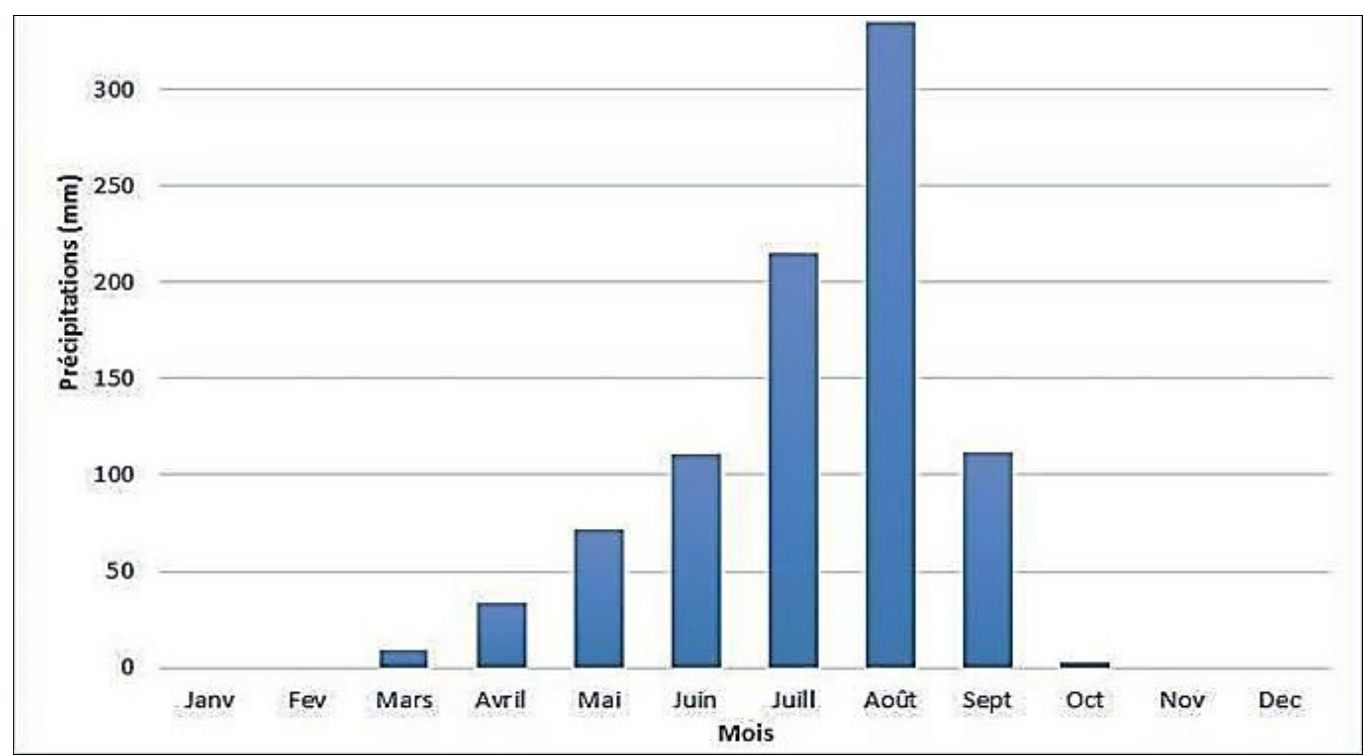

Figure 1 : Diagramme des précipitations du site expérimental de Gampèla en 2016.

Dispositif expérimental: Le dispositif expérimental est un split-plot constitué de trois répétitions. La distance entre les répétitions est de $2 \mathrm{~m}$. Chaque répétition est subdivisée en carrés de rendement ou parcelle élémentaire de 1,6 $\mathrm{m} \times 1,6 \mathrm{~m}$ distants de $1 \mathrm{~m}$ et chaque carré de rendement représente un génotype. Le semis a été réalisé sur trois lignes de $1,6 \mathrm{~m}$ comportant chacune cinq poquets avec des interlignes et des écartements respectifs de $0,8 \mathrm{~m}$ et $0,4 \mathrm{~m}$.

Techniques culturales: L'essai a été conduit entre juillet et novembre 2016. La parcelle a été préalablement labourée au tracteur et nivelée à la daba. Quinze jours après semis, un démariage à un plant par poquet a été effectué. Deux types de fertilisants minéraux ont été utilisés à savoir le NPK
(10-20-20) à la dose de $100 \mathrm{~kg} / \mathrm{ha}$ utilisée comme témoin de référence, $125 \mathrm{~kg} / \mathrm{ha}, 150 \mathrm{~kg} / \mathrm{ha}$ et l'urée perlée $(46 \% \mathrm{~N})$ à la dose de $50 \mathrm{~kg} / \mathrm{ha}$ pour chaque traitement. Le NPK a été apporté sous forme fractionnée respectivement à 14 JAS (jours après semis), 33 JAS et 62 JAS. Le dernier apport de NPK a été combiné avec l'apport d'urée. Un buttage a été ensuite réalisé pour recouvrir l'engrais, protéger les plants contre la verse et maintenir l'humidité du sol.

Collecte des données et analyses statistiques : $\mathrm{Au}$ total neuf variables quantitatives ont été notées. Ces variables sont relatives au nombre de jours 50\% floraison $(\mathbf{5 0} \% \mathrm{NJF})$, au nombre de talles utiles (NTU), à la hauteur de la plante (HPL), au diamètre de la tige principale (DTP), au poids de la panicule principale 
(PPA) et au poids des grains de la panicule principale après égrainage manuel (GPA). Ces paramètres ont été mesurés sur trois plants choisis aléatoirement au stade grains pâteux dans chaque carré de rendement pâteux à l'exception du cycle $50 \%$ floraison. Les paramètres teneur en matière sèche de la paille (TMS), rendement grains à l'hectare (RGR) et rendement paille à l'hectare (RPA) ont été calculés pour tout le carré selon les formules ci-après :

Densité (pieds) $=\left(10000 \mathrm{~m}^{2} \mathrm{X} 15\right) / 2,56 \mathrm{~m}^{2}$

RGR $(\mathrm{kg} / \mathrm{ha})=$ Poids grains du carré $x$ Densité

$\operatorname{RPA}(\mathrm{kg} / \mathrm{ha})=\left(\mathrm{BMS}^{*} 10000 \mathrm{~m}^{2}\right) / 2,56 \mathrm{~m}^{2}$

\section{RESULTATS}

Variation des performances moyennes des génotypes pour chaque niveau de fertilisation minérale : L'analyse des performances moyennes en fonction de chaque niveau de fertilisation minérale montre une très faible différenciation entre les 14 génotypes étudiés. A la dose de $100 \mathrm{~kg} / \mathrm{ha}$ de NPK (Tableau 1), seulement deux des neuf variables étudiées, à savoir le nombre de talles utiles (NTU) et la hauteur de la plante (HPL) discriminent significativement les génotypes au seuil de $5 \%$. La hauteur de la plante a varié de 2,159 m pour le génotype MTC2 à 3,213 m pour le génotype GBI4 et le nombre de talles utiles de 3 (SPI2) à 13 (KBA1 et $\mathrm{YOH} 3$ ). Pour le traitement T1, dose de $125 \mathrm{~kg} / \mathrm{ha}$ de NPK (Tableau 2), seules trois variables à savoir la hauteur de la plante (HPL), le poids de la panicule
TMS $(\%)=((B M S) /(B M F)) \times 100)(B M F:$ biomasse fraiche de la paille, BMS : biomasse sèche de la paille) Afin de mettre en évidence les variations entre les génotypes au sein d'une même dose et les variations de comportement d'un même génotype entre les trois niveaux de fertilisation, des analyses de variance à deux facteurs (fertilisant, génotype) en intégrant le test de séparation des moyennes de Newman-Keuls ont été effectuées à l'aide du logiciel XLSTAT Pro. 2016 en considérant les facteurs génotype et traitement NPK. Les relations entre les caractères ont été déterminées à l'aide du test de corrélation de Pearson.

principale (PPA) et le poids grains de la panicule principale (GPA) discriminent les génotypes au seuil 5 $\%$. La hauteur de la plante a varié de 2,12 m (MTC2) à 2,99 m (GBI4) et le poids de la panicule principale de $117,33 \mathrm{~g}$ (MTC2) à 200,66 g (KBA1). Pour le poids grains de la panicule principale, il a oscillé de $88,03 \mathrm{~g}$ à 171,62 g respectivement pour les génotypes MTC2 et KBA1. Quant à la dose de $150 \mathrm{~kg} / \mathrm{ha}$ de NPK, traitement T2 (Tableau 3), une différence significative entre les performances des génotypes est observée au niveau de deux variables, la hauteur de la plante et le diamètre de la tige principale. Les valeurs extrêmes ont été respectivement de 2,17 m (MTC2) et 3,03 m (GBI4) pour la hauteur et de $1,88 \mathrm{~cm}$ (MTC2) et 2,66 cm (YOU) pour le diamètre de la tige principale. 
Tableau 1 : Performances moyennes des 14 génotypes pour le traitement TR à $100 \mathrm{~kg}$ NPK/ha

\begin{tabular}{|c|c|c|c|c|c|c|c|c|c|}
\hline Génot. & $\begin{array}{l}50 \% \text { NJF } \\
\text { jours }\end{array}$ & NTU & $\begin{array}{l}\text { HPL } \\
M\end{array}$ & $\begin{array}{l}\text { DTP } \\
\mathrm{cm}\end{array}$ & $\begin{array}{l}\text { PPA } \\
\text { G }\end{array}$ & $\begin{array}{l}\text { GPA } \\
\text { G }\end{array}$ & $\begin{array}{l}\text { TMS } \\
\%\end{array}$ & $\begin{array}{l}\text { RGR/ha } \\
\text { kg }\end{array}$ & $\begin{array}{l}\text { RPA/ha } \\
\mathrm{Kg}\end{array}$ \\
\hline BKB1 & $78,67 a$ & $9,67 a$ & $2,77 b$ & $2,78 a$ & $165,24 a$ & $135,61 a$ & $42,17 a$ & $7449,12 a$ & $11309,90 a$ \\
\hline SBR7 & $78,33 a$ & $12,00 \mathrm{a}$ & $2,74 b$ & $3,62 \mathrm{a}$ & $148,45 a$ & $125,43 a$ & $53,94 a$ & $8279,84 a$ & $12683,59 \mathrm{a}$ \\
\hline KBA1 & $81,00 \mathrm{a}$ & $\underline{13,33 a}$ & $2,75 b$ & $\overline{2,60 a}$ & $165,96 a$ & $138,12 a$ & $\overline{47,03 a}$ & $\overline{7424,38 a}$ & $\overline{11139,32 a}$ \\
\hline PBO5 & $79,00 \mathrm{a}$ & $\overline{11,67 a}$ & $2,67 b$ & $2,56 a$ & $134,93 a$ & $108,50 a$ & $48,59 a$ & $7442,61 a$ & $11223,96 a$ \\
\hline YOH3 & $77,33 a$ & $\underline{13,33 a}$ & $2,81 b$ & $2,45 a$ & $130,97 a$ & $107,84 a$ & $53,40 a$ & $7061,11 a$ & $12161,46 a$ \\
\hline SBR1 & $80,00 a$ & $9,67 a$ & $2,57 b$ & $2,72 a$ & $160,31 a$ & $121,22 a$ & $42,22 a$ & $6704,34 a$ & $10742,19 a$ \\
\hline SPI2 & $82,33 a$ & $3,00 a$ & $2,80 \mathrm{~b}$ & $2,83 a$ & $189,52 a$ & $156,29 a$ & $39,28 a$ & $6029,09 a$ & $7852,87 a$ \\
\hline GBI4 & $79,67 a$ & $5,33 a$ & $3,21 a$ & $2,51 a$ & $190,33 a$ & $\overline{141,53 a}$ & $43,12 a$ & $6385,34 a$ & $10048,18 a$ \\
\hline MBO7 & $80,00 \mathrm{a}$ & $8,33 a$ & $\overline{2,53 b}$ & $2,55 a$ & $\overline{160,22 a}$ & $127,29 a$ & $47,37 a$ & $4365,83 a$ & $4881,51 a$ \\
\hline YOU5 & $76,67 a$ & $12,00 \mathrm{a}$ & $2,59 \mathrm{~b}$ & $2,34 a$ & $123,81 a$ & $98,07 a$ & $45,49 a$ & $5969,98 a$ & $8716,15 a$ \\
\hline PGO3 & $80,67 a$ & $4,67 a$ & $2,56 \mathrm{~b}$ & $2,64 a$ & $154,59 a$ & $113,71 a$ & $39,97 a$ & $5563,73 a$ & $7843,75 a$ \\
\hline BK01 & $80,67 a$ & $5,67 a$ & $2,59 \mathrm{~b}$ & $2,20 a$ & $137,63 a$ & $110,02 a$ & $39,24 a$ & $5390,56 a$ & $6009,12 a$ \\
\hline BZI1 & $82,00 \mathrm{a}$ & $6,33 a$ & $2,49 b$ & $\underline{2,19 a}$ & $112,00 \mathrm{a}$ & $88,34 a$ & $39,76 a$ & $4837,18 a$ & $7011,72 a$ \\
\hline MTC2 & $78,33 a$ & $4,33 a$ & $2,16 \mathrm{c}$ & $\overline{2,30 a}$ & $105,28 \mathrm{a}$ & $79,36 \mathrm{a}$ & $42,10 a$ & $3964,79 a$ & $6437,50 a$ \\
\hline Moy. & $79,62 a$ & $9 a$ & $2,66 a$ & $2,59 a$ & $148,52 a$ & $117,95 a$ & $44,55 a$ & $6204,85 a$ & $9147,23 a$ \\
\hline $\operatorname{Pr}>\mathrm{F}$ & 0,065 & 0,018 & 0,000 & 0,425 & 0,062 & 0,083 & 0,866 & 0,126 & 0,087 \\
\hline Signif. & Non & Oui & Oui & Non & Non & Non & Non & Non & Non \\
\hline
\end{tabular}

Tableau 2 : Performances moyennes des 14 génotypes pour le traitement T1 à 125kg NPK/ha

\begin{tabular}{|c|c|c|c|c|c|c|c|c|c|}
\hline Génot. & $\begin{array}{l}50 \% \text { NJF } \\
\text { jours }\end{array}$ & NTU & $\begin{array}{l}\text { HPL } \\
M\end{array}$ & $\begin{array}{l}\text { DTP } \\
\mathrm{cm}\end{array}$ & $\begin{array}{l}\text { PPA } \\
\text { G }\end{array}$ & $\begin{array}{l}\text { GPA } \\
\text { G }\end{array}$ & $\begin{array}{l}\text { TMS } \\
\%\end{array}$ & $\begin{array}{l}\text { RGR/ha } \\
\mathrm{kg}\end{array}$ & $\begin{array}{l}\text { RPA/ha } \\
\mathrm{kg}\end{array}$ \\
\hline KBA1 & $81,67 a$ & $7,67 a$ & $2,73 a$ & $2,63 a$ & $200,66 a$ & $171,62 \mathrm{a}$ & $40,67 a$ & $8300,68 a$ & $10000,00 \mathrm{a}$ \\
\hline SPI2 & $81,33 a$ & $7,67 a$ & $2,79 a$ & $\overline{2,60 a}$ & $\overline{191,11 a b}$ & $\overline{161,48 a b}$ & $43,99 a$ & $\overline{6839,76 a}$ & $\overline{11165,37 a}$ \\
\hline YOH3 & $81,00 \mathrm{a}$ & $11,00 \mathrm{a}$ & $2,73 a$ & $2,39 a$ & $151,98 a b c$ & 126,63abcd & $42,43 a$ & $7469,96 a$ & $\overline{10786,46 a}$ \\
\hline BZI1 & $79,33 a$ & $\overline{10,67 a}$ & $2,78 a$ & $2,29 a$ & $171,01 a b c$ & $143,77 a b c$ & $37,34 a$ & $8130,10 a$ & $9470,05 a$ \\
\hline PGO3 & $80,67 a$ & $8,00 a$ & $2,55 a b$ & $2,73 a$ & $161,27 a b c$ & $125,74 a b c d$ & $42,30 \mathrm{a}$ & $6955,64 a$ & $9250,00 a$ \\
\hline SBR7 & $80,67 a$ & $8,00 a$ & $2,82 a$ & $2,42 a$ & $159,32 a b c$ & 133,01abcd & $38,49 a$ & $6413,98 a$ & $8752,60 a$ \\
\hline GBI4 & $82,00 \mathrm{a}$ & $7,33 a$ & $2,99 a$ & $2,29 a$ & $159,17 a b c$ & $126,57 \mathrm{abcd}$ & $38,89 a$ & $7081,94 a$ & $10270,8 a$ \\
\hline SBR1 & $82,33 a$ & $4,00 a$ & $\overline{2,43 a b}$ & $2,60 a$ & $192,58 \mathrm{ab}$ & $149,03 a b c$ & $36,73 a$ & $6482,99 a$ & $8434,90 a$ \\
\hline BKB1 & $80,00 a$ & $5,33 a$ & $2,72 a$ & $2,53 a$ & $168,71 \mathrm{abc}$ & $140,09 \mathrm{abc}$ & $31,86 a$ & $5755,14 a$ & $6739,58 a$ \\
\hline
\end{tabular}




\begin{tabular}{|c|c|c|c|c|c|c|c|c|c|}
\hline BK01 & $80,67 a$ & $6,67 a$ & $2,45 a b$ & $2,61 a$ & $141,16 a b c$ & $109,78 \mathrm{bcd}$ & $49,75 a$ & $6338,46 a$ & $8654,95 a$ \\
\hline YOU5 & $80,67 a$ & $9,00 \mathrm{a}$ & $2,55 a b$ & $2,32 a$ & $156,04 a b c$ & $133,12 \mathrm{abcd}$ & $39,50 a$ & $6665,28 a$ & $7460,94 a$ \\
\hline PBO5 & $82,00 \mathrm{a}$ & $5,00 a$ & $2,56 a b$ & $2,51 \mathrm{a}$ & $134,40 \mathrm{bc}$ & $103,68 \mathrm{~cd}$ & $44,16 \mathrm{a}$ & $4611,92 a$ & $6548,18 a$ \\
\hline MBO7 & $80,00 a$ & $9,67 a$ & $2,74 a$ & $2,33 a$ & $153,47 a b c$ & $127,02 \mathrm{abcd}$ & $33,18 a$ & $4899,68 a$ & $5641,93 a$ \\
\hline MTC2 & $78,33 a$ & $\underline{2,33 a}$ & $\underline{2,12 b}$ & $\underline{2,25 a}$ & $117,33 \mathrm{c}$ & $88,03 d$ & $\underline{37,80 \mathrm{a}}$ & $3415,32 a$ & $\underline{4154,95 a}$ \\
\hline Moy. & $80,76 a$ & $8 a$ & $2,64 a$ & $2,46 a$ & $161,30 a$ & $131,40 a$ & $39,79 b$ & $6382,92 a$ & $8380,77 a$ \\
\hline $\operatorname{Pr}>\mathrm{F}$ & 0,813 & 0,569 & 0,002 & 0,087 & 0,002 & 0,001 & 0,346 & 0,606 & 0,526 \\
\hline Signif. & Non & Non & Oui & Non & Oui & Oui & Non & Non & Non \\
\hline
\end{tabular}

Moy. : Moyenne, 50 \% NJF : nombre de jour $50 \%$ floraison, NTU : nombre de talles utiles, HPL : hauteur de la plante, , PPA : poids de la panicule principale, GPA :poids grains de la panicule principale, RGR rendement grain à l'hectare, RPA : rendement paille à l'hectare, TMS : teneur en matière sèche de la paille, Pr : probabilité du $F$ de Fischer, a, b, $c$ : les valeurs suivies par les mêmes lettres ne sont pas significativement différentes, ${ }^{*}$ : différence significative à $5 \%$, ${ }^{* *}:$ différence hautement significative à $5 \%$, ${ }^{* * *}:$ très hautement significative à $5 \%$, ns : différence non significative.

Tableau 3 : Performances moyennes des 14 génotypes pour le traitement T2 à 150kg NPK/ha

\begin{tabular}{|c|c|c|c|c|c|c|c|c|c|}
\hline Génot. & $\begin{array}{l}\mathbf{5 0} \% \mathrm{NJF} \\
\text { jours }\end{array}$ & NTU & $\begin{array}{l}\text { HPL } \\
M\end{array}$ & $\begin{array}{l}\text { DTP } \\
\text { Cm }\end{array}$ & $\begin{array}{l}\text { PPA } \\
\text { G }\end{array}$ & $\begin{array}{l}\text { GPA } \\
\text { G }\end{array}$ & $\begin{array}{l}\text { TMS } \\
\%\end{array}$ & $\begin{array}{l}\text { RGR/ha } \\
\text { kg }\end{array}$ & $\begin{array}{l}\text { RPA/ha } \\
\text { kg }\end{array}$ \\
\hline PBO5 & $80,33 a$ & $9 a$ & $2,79 a b c$ & $2,60 a$ & $179,91 \mathrm{a}$ & $148,31 \mathrm{a}$ & $41,79 a$ & $8455,62 a$ & $10522,14 a$ \\
\hline KBA1 & $80,00 a$ & $11 a$ & $2,63 a b c$ & $2,47 a$ & $\overline{173,10 a}$ & $\overline{143,53 a}$ & $\underline{47,07 a}$ & $7490,79 a$ & $10683,59 a$ \\
\hline SBR7 & $78,67 a$ & $13 a$ & $2,78 a b c$ & $2,50 a$ & $159,17 a$ & $126,57 a$ & $\overline{37,66 a}$ & $8969,94 a$ & $10359,38 a$ \\
\hline GBI4 & $80,33 a$ & $\overline{9 a}$ & $3,03 a$ & $2,45 a$ & $152,96 \mathrm{a}$ & $117,98 a$ & $49,72 a$ & $\overline{6851,48 a}$ & $12515,63 \mathrm{a}$ \\
\hline BKB1 & $80,67 a$ & $5 a$ & $\overline{2,68 a b c}$ & $2,48 a$ & $164,31 \mathrm{a}$ & $134,69 a$ & $45,41 a$ & $6696,53 a$ & $9037,76 a$ \\
\hline SPI2 & $80,00 a$ & $8 a$ & $2,93 a b$ & $2,51 a$ & $165,11 a$ & $141,14 a$ & $35,24 a$ & $7943,91 a$ & $9424,48 a$ \\
\hline SBR1 & $79,00 \mathrm{a}$ & $7 a$ & $2,65 a b c$ & $2,36 a$ & $159,46 a$ & $130,02 a$ & $43,65 a$ & $6679,60 a$ & $10563,80 a$ \\
\hline YOU5 & $79,00 \mathrm{a}$ & $11 a$ & $2,44 \mathrm{~cd}$ & $2,66 a$ & $164,61 a$ & $134,90 \mathrm{a}$ & $38,65 a$ & $5981,69 a$ & $7559,90 \mathrm{a}$ \\
\hline PGO3 & $82,67 a$ & $4 a$ & $2,54 b c$ & $\overline{2,58 a}$ & $178,62 a$ & $140,46 a$ & $36,62 a$ & $5005,14 a$ & $6662,76 a$ \\
\hline BZI1 & $79,67 a$ & $9 a$ & $2,71 \mathrm{abc}$ & $2,24 a$ & $158,10 a$ & $132,93 a$ & $34,21 a$ & $7012,93 a$ & $8282,55 a$ \\
\hline MBO7 & $81,33 a$ & $8 a$ & $2,72 a b c$ & $2,46 a$ & $156,29 a$ & $128,81 a$ & $40,75 a$ & $5368,94 a$ & $6632,81 a$ \\
\hline BK01 & $80,00 a$ & $8 a$ & $2,51 b c$ & $2,28 a$ & $153,67 a$ & $123,40 a$ & $46,43 a$ & $5542,90 a$ & $8854,17 a$ \\
\hline YOH3 & $83,67 a$ & $4 a$ & $2,57 a b c$ & $2,22 a$ & $143,41 a$ & $113,16 a$ & $39,40 a$ & $3699,17 a$ & $6037,76 a$ \\
\hline MTC2 & $81,00 a$ & $6 a$ & $2,17 d$ & $1,88 \mathrm{~b}$ & $107,36 \mathrm{a}$ & $86,46 a$ & $36,40 \mathrm{a}$ & $3397,09 a$ & $4888,02 a$ \\
\hline Moy. & $80,45 a$ & $8 a$ & $2,65 a$ & $2,41 a$ & $158,29 a$ & $128,74 a$ & $40,93 a b$ & $6363,98 a$ & $8716,05 a$ \\
\hline $\operatorname{Pr}>\mathrm{F}$ & 0,264 & 0,485 & 0,000 & 0,005 & 0,220 & 0,226 & 0,287 & 0,105 & 0,245 \\
\hline Signif. & Non & Non & Oui & Oui & Non & Non & Non & Non & Non \\
\hline
\end{tabular}

Moy. :Moyenne, 50 \% NJF : nombre de jour 50 \% floraison, NTU : nombre de talles utiles, HPL : hauteur de la plante, , PPA: poids de la panicule principale, GPA :poids grains de la panicule principale, , RGR rendement grain à l'hectare, RPA : rendement paille à l'hectare, TMS : teneur en matière sèche de la paille, Pr : probabilité du F de Fischer, a, b, $c$ : les valeurs suivies par les mêmes lettres ne sont pas significativement différentes, ${ }^{*}:$ différence significative à $5 \%$, ${ }^{* *}:$ différence hautement significative à $5 \%$, ${ }^{* *}:$ différence très hautement significative à $5 \%$, ns :différence non significative. 
Effet de la fertilisation sur les paramètres agromorphologiques: La comparaison des moyennes inter-traitements a révélé qu'à l'exception de la teneur en matière sèche de la paille (figure 2) qui a présenté une différence significative au seuil de $5 \%$, l'effet dose de NPK n'a pas influencé les autres paramètres agromorphologiques (Tableau 4).

Tableau 4 : Comportement des génotypes en fonction des trois niveaux de fertilisation

\begin{tabular}{llllllllll}
\hline Trt & $\begin{array}{l}\mathbf{5 0} \% \text { NJF } \\
\text { (jr) }\end{array}$ & NTU & $\begin{array}{lllll}\text { HPL } \\
\mathbf{m}\end{array}$ & $\begin{array}{l}\text { DTP } \\
\text { cm }\end{array}$ & $\begin{array}{l}\text { PPA } \\
\mathbf{g}\end{array}$ & $\begin{array}{l}\text { GPA } \\
\mathbf{g}\end{array}$ & $\begin{array}{l}\text { TMS } \\
\%\end{array}$ & $\begin{array}{l}\text { RGR/ha } \\
\mathbf{K g}\end{array}$ & $\begin{array}{l}\text { RPA/ha } \\
\mathbf{k g}\end{array}$ \\
\hline T1 & $80,76 \mathrm{a}$ & $8 \mathrm{a}$ & $2,64 \mathrm{a}$ & $2,46 \mathrm{a}$ & $161,30 \mathrm{a}$ & $131,40 \mathrm{a}$ & $39,79 \underline{\mathbf{b}}$ & $6382,92 \mathrm{a}$ & $8380,77 \mathrm{a}$ \\
TR & $79,62 \mathrm{a}$ & $9 \mathrm{a}$ & $2,66 \mathrm{a}$ & $2,59 \mathrm{a}$ & $148,52 \mathrm{a}$ & $117,95 \mathrm{a}$ & $44,55 \underline{\mathbf{a}}$ & $6204,85 \mathrm{a}$ & $9147,23 \mathrm{a}$ \\
T2 & $80,45 \mathrm{a}$ & $8 \mathrm{a}$ & $2,65 \mathrm{a}$ & $2,41 \mathrm{a}$ & $158,29 \mathrm{a}$ & $128,74 \mathrm{a}$ & $40,93 \underline{\mathbf{a b}}$ & $6363,98 \mathrm{a}$ & $8716,05 \mathrm{a}$ \\
\hline Pr>F & $\mathbf{0 , 1}$ & $\mathbf{0 , 6 5}$ & $\mathbf{0 , 9 7}$ & $\mathbf{0 , 1 5}$ & $\mathbf{0 , 3 0}$ & $\mathbf{0 , 1 8}$ & $\mathbf{0 , 0 3}$ & $\mathbf{0 , 9 4}$ & $\mathbf{0 , 6 7}$ \\
Signif. & Non & Non & Non & Non & Non & Non & Oui & Non & Non \\
\hline
\end{tabular}

Trt :traitement; 50 \% NJF : nombre de jour 50 \% floraison, NTU : nombre de talles utiles, HPL : hauteur de la plante, , PPA: poids de la panicule principale, GPA :poids grains de la panicule principale, , RGR rendement grain à l'hectare, RPA : rendement paille à l'hectare, TMS : teneur en matière sèche de la paille, $\mathrm{Pr}$ : probabilité du $\mathrm{F}$ de Fischer, $a, b, c$ : les valeurs suivies par les mêmes lettres ne sont pas significativement différentes, ${ }^{*}$ : différence significative à $5 \%,{ }^{* *}$ : différence hautement significative à 5 $\%,{ }^{* * *}$ : différence très hautement significative, ns :différence non significative.

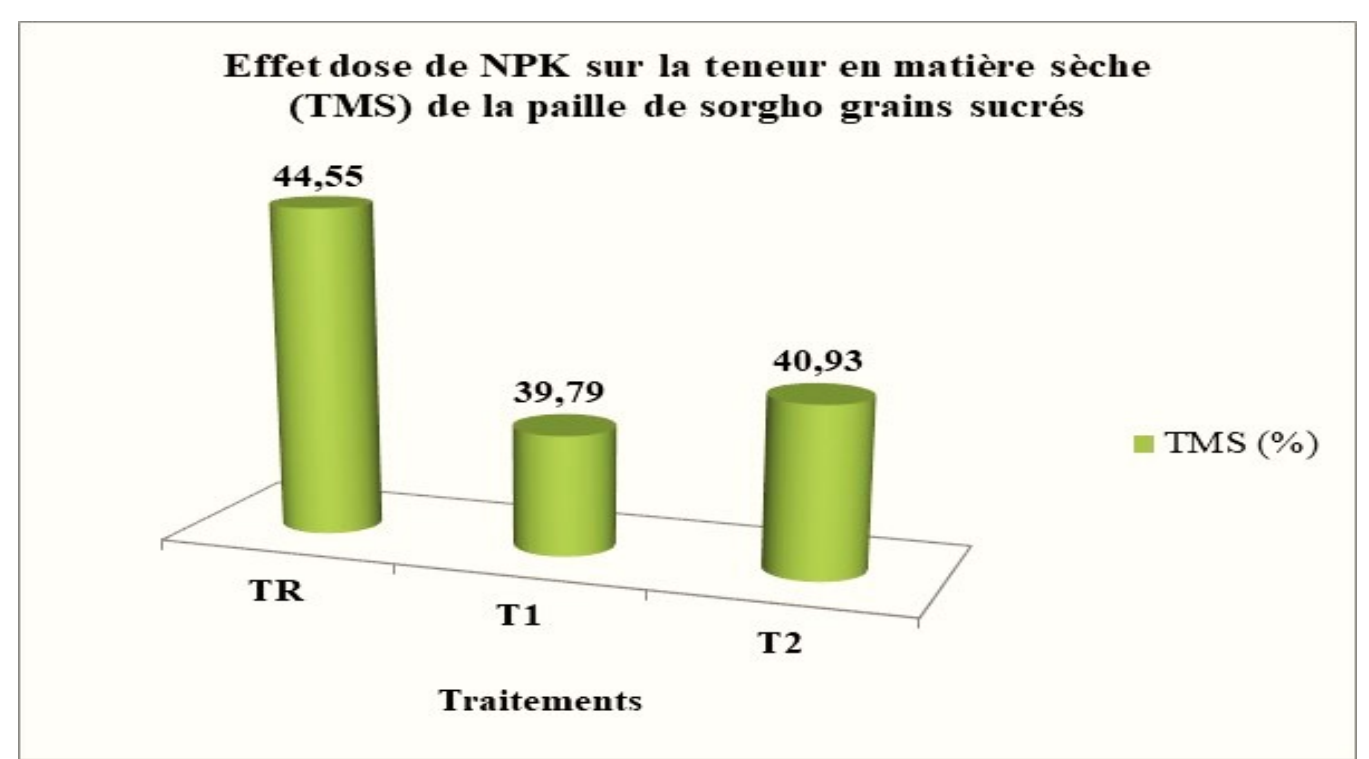

Figure 2 : Effet de la fertilisation minérale (NPK) sur la teneur en matière sèche de la paille du sorgho grains sucrés.

Effet de la fertilisation minérale sur les performances de chaque génotype : La performance des génotypes a varié en fonction des traitements (figure 3). Ainsi, pour le nombre de talles utiles (figure 3-a), les génotypes KBA1 et $\mathrm{YOH} 3$ ont présenté un nombre de talles élevé $(13,33)$ à la dose de $100 \mathrm{~kg}$ NPK/ha et le génotype MTC2 le plus petit nombre $(2,12)$ à la dose de $125 \mathrm{~kg} \mathrm{NPK} / \mathrm{ha}$. Pour la hauteur de la plante, tous les génotypes se sont bien exprimés dans le traitement TR (100 kg NPK/ha). La plus grande taille $(3,21 \mathrm{~m})$ a été enregistrée à la dose TR avec le génotype GBI4 et la plus petite $(2,12 \mathrm{~m})$ à T1 avec le génotype MTC2 (Figure 3-b). En ce qui concerne le poids de la panicule principale (PPA) et le poids grains de la panicule principale (GPA), KBA1 a présenté les plus fortes valeurs de 200,66 g (figure 3-c) et de 171,62 g (figure 3-d), au traitement T1 (125 kg NPK/ha) et le génotype MTC2 a les plus faibles valeurs de 105,28 g et $79,36 \mathrm{~g}$ pour l'ensemble des traitements. Pour le rendement à l'hectare, le génotype SBR7 a présenté le meilleur rendement grains avec $8279,84 \mathrm{~kg} / \mathrm{ha}$ (figure 3 -e) et le meilleur rendement paille avec $12683,59 \mathrm{~kg}$ 
(figure 3-f) respectivement à 125 et $100 \mathrm{~kg} \mathrm{NPK/ha.} \mathrm{Le}$ génotype GBI4 a également un bon rendement paille $(12515,63 \mathrm{~kg} / \mathrm{ha})$ à la dose de $125 \mathrm{~kg} \mathrm{NPK} / \mathrm{ha}$. Par contre, les plus faibles rendements grains et paille ont été obtenus par le génotype MTC2 avec des valeurs respectives de $3397,09 \mathrm{~kg} / \mathrm{h}$ a et 4154,95 kg/ha. Malgré cette faible variabilité au sein des génotypes dans chaque traitement, les génotypes BKB1, SBR7, KBA1, PBO5, YOH3, SBR1 et GBI4 ont présenté des rendements grains et paille supérieurs à la moyenne $\mathrm{du}$ traitement soit $6204,85 \mathrm{~kg} / \mathrm{ha}$ pour le grain et 9147,23 $\mathrm{kg} / \mathrm{ha}$ pour la paille à la dose TR. A T1, neuf (9) génotypes à savoir SBR7, KBA1, PGO3, YOH3, SBR1, GBI4, BZI1, SPI2 et YOU5 ont montré des rendements supérieurs aux rendements moyens en grains et en paille du traitement respectivement égal à 6382,92 $\mathrm{kg} / \mathrm{ha}$ et $8380,77 \mathrm{~kg} / \mathrm{ha}$. La dernière dose $\mathrm{T} 2$, a par contre montré que SBR7, KBA1, PBO5, SBR1, GBI4, BZI1, SPI2 et BKB1 affichent des rendements grains et paille plus importants que le rendement moyen en grains $(6363,98 \mathrm{~kg} / \mathrm{ha})$ et en paille $(8716,05 \mathrm{~kg} / \mathrm{ha}) \mathrm{du}$ traitement.

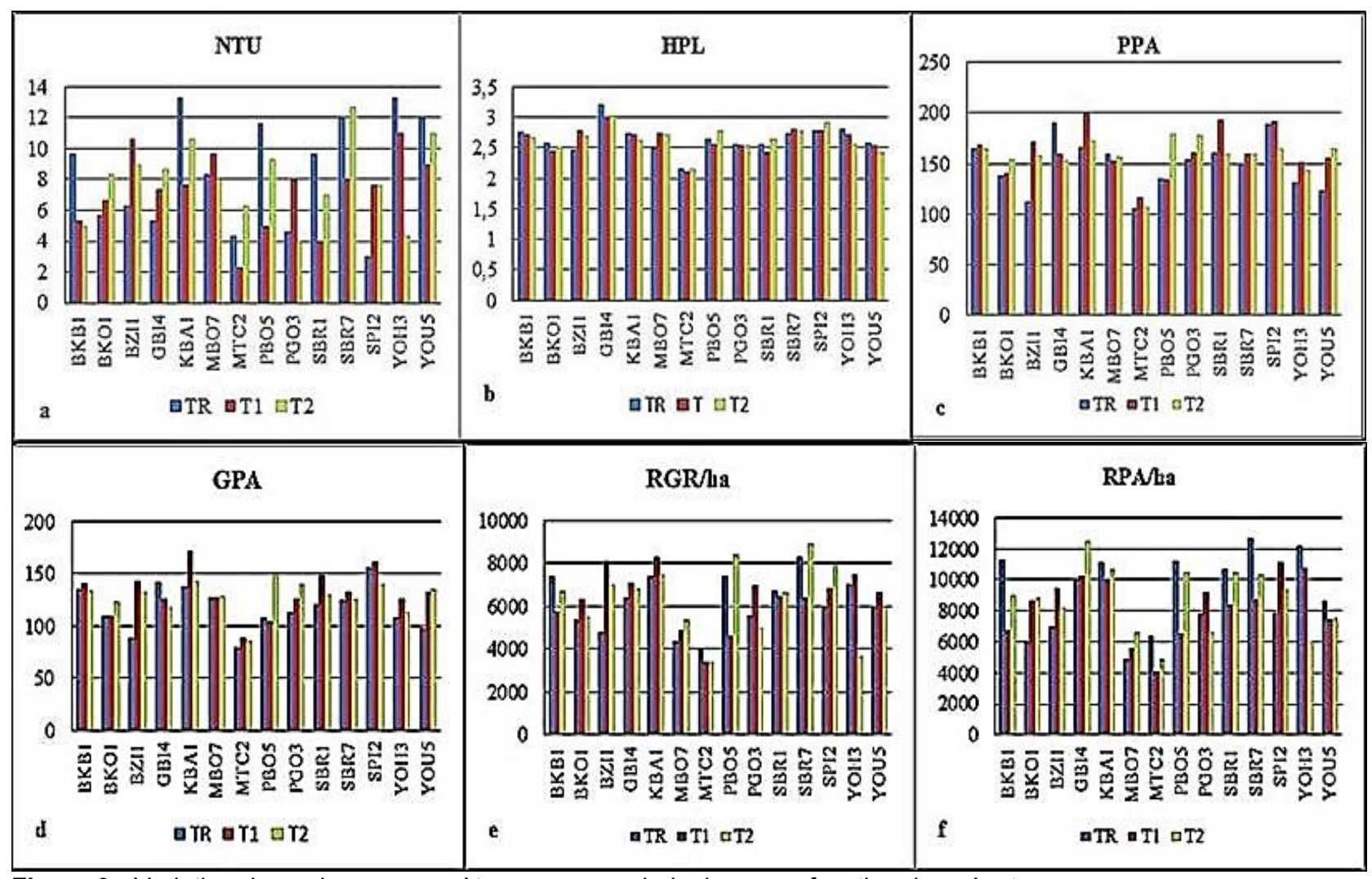

Figure 3 : Variation de quelques paramètres agro-morphologiques en fonction des génotypes

$a:$ nombre de talles utiles, $b:$ hauteur de la plante, $c$ : poids de la panicule principale, $d:$ poids grains de la panicule principale, e rendement grain à l'hectare, $f$ : rendement paille à l'hectare).

TR (100 kg NPK/ha), T1 (125 kg NPK/ha) et T2 (150 kg NPK/ha)

Relations entre les caractères étudiés : La matrice de corrélation (Tableau 5) indique plusieurs corrélations significatives, fortes et positives. Ainsi, la corrélation est positive entre le poids grains de la panicule principale (GPA) et chacune des variables, hauteur de la plante (HPL), diamètre de la tige principale (DTP), poids de la panicule principale (PPA). Des corrélations positives sont également observées entre le nombre de talles utiles (NTU) et chacun des paramètres rendement grains (RGR) et rendement paille (RPA) et entre les rendements grains (RGR) et le rendement paille (RPA) à $(r=0,84)$. II en est de même pour le poids de la panicule principale (PPA) et le poids des grains de la panicule principale (GPA) $(r=0,970)$. 
Tableau 5 : Matrice de corrélation entre les caractères étudiés

\begin{tabular}{|c|c|c|c|c|c|c|c|c|}
\hline Variables & $50 \%$ NJF & NTU & HPL & DTP & & GPA & TMS & RGR/ha \\
\hline NTU & $-0,56^{* * *}$ & & & & & & & \\
\hline HPL & 0,03 & 0,29 & & & & & & \\
\hline DTP & $-0,11$ & 0,20 & 0,26 & & & & & \\
\hline PPA & $0,32^{*}$ & $-0,04$ & $0,55^{\star * *}$ & $0,42^{* *}$ & & & & \\
\hline GPA & 0,28 & 0,07 & $0,56^{* *}$ & $0,40^{* *}$ & $0,97^{* * *}$ & & & \\
\hline TMS & $-0,31^{*}$ & $0,39^{*}$ & 0,12 & 0,40 ** & $-0,15$ & $-0,15$ & & \\
\hline RGR/ha & $-0,22$ & $0,61^{* * *}$ & $0,63^{* * *}$ & 0,46 ** & $0,53^{* * *}$ & $0,59^{* * *}$ & 0,23 & \\
\hline RPA/ha & $-0,26$ & $0,60^{* * *}$ & $0,62^{* * *}$ & 0,48 ** & $0,36^{*}$ & $0,38^{*}$ & $0,55^{* * *}$ & $0,84^{* * *}$ \\
\hline
\end{tabular}

${ }^{*}$ : Différence significative à $5 \%,{ }^{* *}$ : différence significative à $1 \%,{ }^{* * *}$ : hautement significative, ns : différence non significative

\section{DISCUSSION}

La faible variabilité observée entre les 14 génotypes à l'intérieur d'un même traitement pourrait s'expliquer par la proximité génétique des génotypes utilisés, leur permettant ainsi de s'adapter de la même façon aux conditions physico-chimiques du milieu. Ces résultats diffèrent de ceux de Nebié et al., (2012), Sawadogo et al., (2014a), Sawadogo et al., (2014b) et Tiendrebéogo et al. (2018) sur respectivement des sorghos grains sucrés du Centre Nord, du Nord du Burkina et d'une collection nationale. En effet, les 14 génotypes sont issus de la collection nationale constituée des accessions de toutes les zones agro-climatiques du pays. Ils ont été sélectionnés sur la base de leur performance agro-morphologique et de leur productivité en grains et en fourrage élevées (Tiendrebeogo et al, 2018). Les rendements grains potentiels à l'hectare de $8279,84 \mathrm{~kg} / \mathrm{ha}$ obtenus pour le meilleur génotype (SBR7) et $3964,79 \mathrm{~kg} / \mathrm{ha}$ pour le moins productif (MTC2) à la dose de $100 \mathrm{~kg} \mathrm{NPK/ha} \mathrm{sont} \mathrm{largement}$ supérieurs au rendement actuel du sorgho grains au Burkina qui est de $875 \mathrm{~kg} / \mathrm{ha}$ pour le sorgho blanc et $1104 \mathrm{~kg} / \mathrm{ha}$ pour le sorgho rouge (DGESS/MAAH, 2017). Cette grande différence se justifierait par le fait que dans le cas du sorgho grains sucrés, les grains ont été récoltés au stade pâteux, période pendant laquelle la teneur en eau des grains varie entre 26,72 et 52,44 $\%$ (Tiendrebeogo et al., 2018). Cependant, pour le rendement paille, la valeur moyenne de 9147,23 kg/ha obtenue est très proche de celle de Kondombo, 2001 sur des lignées recombinantes de sorgho à double usage (Sariaso $10 \times$ pelego) et de celle de Ndiaye et al., 2018 sur des génotypes de sorgho issus de régions divers d'Afrique de l'Ouest et Centrale, reconnus pour leurs performances agronomiques. Les sorghos grains sucrés présenteraient donc des possibilités de production à double usage (production en grains et en fourrage). Cela est confirmée par l'existence d'une forte corrélation positive entre le rendement grains (RGR/ha) et le rendement paille (RPA/ha). De plus, la forte corrélation positive entre le rendement grains (RGR/ha) et le nombre de talles suggère que l'augmentation du nombre de talles entrainerait une augmentation du nombre de panicules récoltées influençant ainsi le rendement. Des résultats similaires ont été rapportés par Chahredine et Benderradji (2019) sur des variétés de blé tendre (Triticum aestium L.) en Algérie. En outre, cette possibilité de sélection du sorgho grains sucrés pour un usage mixte est également traduite par les corrélations positives entre les paramètres de développement végétatif de la plante que sont la hauteur de la plante $(\mathrm{HPL})$, le diamètre de la tige principale (DTP) et les composantes du rendement telles que le poids grains de la panicule principale (GPA), le poids de la panicule principale (PPA). Des résultats similaires ont été observés par Innes et Bakwell (1981) et Gate et al. (1993) sur le blé. Selon ces auteurs, il y aurait une meilleure remobilisation possible des assimilât de la tige vers les grains permettant ainsi aux grains d'avoir de meilleurs poids. Par ailleurs, les valeurs moyennes du nombre de talles utiles (NTU), du diamètre de la tige principale (DTP) et du poids grains de la panicule principale GPA) à la dose de $100 \mathrm{~kg} \mathrm{NPK} /$ ha sont élevées comparativement à celles de Sawadogo (2015) sur des accessions de sorghos grains sucrés. Ces différences pourraient être attribuées au fait que ce sont les meilleurs génotypes qui ont été retenus pour la présente étude. En effet, les critères de sélection des génotypes ont pris en compte ces différents paramètres qui contribuent énormément à la hausse du rendement grains et fourrage. L'effet significatif de la variation de la dose d'engrais sur uniquement la teneur en matière sèche de la paille (TMS) pourrait s'expliquerait par le fait qu'il y a une dose optimale pour laquelle il y a une bonne accumulation des éléments minéraux par la plante qui se traduit par un bon développement végétatif et la 
production d'une biomasse considérable. En effet, Cattan et al., (2001) chez le maïs et Bekalu et al. (2016) sur le blé ont indiqué que ces cultures possèderaient un optimum de fertilisation azotée qui rehausse considérablement le rendement en biomasse. De ce fait, une dose qui dépasse l'optimum contribue à baisser ce rendement. Ainsi, chez le blé l'engrais azoté à la dose de $69 \mathrm{~kg} / \mathrm{ha}$ contribue à une augmentation de la biomasse du blé de $21,1 \%$ à $24,3 \%$ contrairement à la dose de $23 \mathrm{~kg} / \mathrm{ha}$ et celle de $92 \mathrm{~kg} / \mathrm{ha}$. Une faible influence des facteurs environnementaux sur l'expression des performances d'accessions de sorgho pluvial du Caméroun a également été signalée (Danbe et al., 2018). L'absence d'effet significatif de la variation de la dose de NPK sur la plupart des caractères pourrait s'expliquer par le fait que la dose minimale administrée de $100 \mathrm{~kg} \mathrm{NPK/ha}$ est optimale pour

\section{CONCLUSION}

L'étude a mis en évidence les performances de production des 14 génotypes de sorgho grains sucrés en fonction du niveau de fertilisation minérale NPK. Des neuf variables étudiées, seule la teneur en matière sèche (TMS) a été significativement influencée par l'effet traitement. Dans l'optique de concilier forte productivité, faible consommation d'intrants et réduction des couts liés à la production, le traitement TR $(100 \mathrm{~kg}$ de NPK/ha) pourrait être recommandé comme dose optimale pour les itinéraires techniques de la production

\section{REMERCIEMENT}

Les auteurs traduisent toute leur reconnaissance aux membres de l'Equipe Génétique et Amélioration des Plantes du Laboratoires Biosciences (Université Joseph $\mathrm{KI}$-ZERBO), les étudiants (es) de Master et le

\section{REFERENCES BIBLIOGRAPHIQUES}

Bekalu A. and Mamo M., 2016. Effect of the rate of nfertilizer application on growth and yield of wheat (Triticum aestivum L.) at Chencha, Southern Ethiopia. International Journal of Plant, Animal and Environmental Sciences, pp 168-175.

Cattan P., Letourmy P., Zagre B., Minougou A., Compaore E., 2001. Rendement de l'arachide et du sorgho en rotation sous différents itinéraires techniques au Burkina Faso. Cahiers Agricultures pp 159-172.

Chahredine Sadek et Benderradji Laid, 2019. Evaluation du progrès génétique de quinze (15) variétés de blé tendre (Triticum aestivum l'expression maximale de ces paramètres. Elle aurait été suffisante pour couvrir les besoins minéraux de la culture. Cette dose de $100 \mathrm{Kg} \mathrm{NPK} / \mathrm{ha}$ recommandée pour la production du sorgho grains pourrait également être retenue pour la production du sorgho grains sucrés double usage. L'absence de l'effet fertilisation minérale sur la variable phénologique (nombre de jours $50 \%$ NJF) est similaire à celle obtenue par Hani (2000) sur quatre cultivars de sorgho fourrager du Soudan. Ceci pourrait être attribué au fait que la floraison est un caractère génotypique. En effet, Sawadogo (2015) avait rapporté une héritabilité au sens large $\left(\mathrm{H}^{2}\right)$ élevé de l'ordre de $86,05 \%$ pour ce caractère sur un échantillon de 97 accessions de sorghos grains sucrés. Ce caractère serait beaucoup plus influencé par le génotype que l'environnement.

de sorgho grains sucrés et les cinq génotypes KBA1, GBI4, BKB1, SBR1 et SBR7 pourraient être retenus comme parents géniteurs élites pour la création de variétés de sorgho grains sucrés à double usage. Des essais multi-locaux et des analyses chimiques des grains et de la paille pour recueillir des informations sur les qualités nutritionnelles des grains pour l'homme et de la paille pour les animaux pourraient être envisagées pour compléter les résultats de cette étude.

technicien pour leur appui sans faille dans la réalisation de ce travail. Nos remerciements vont également aux Fondations Rosa Luxembourg et Harald Breuer pour l'appui financier dans la réalisation de ce travail.

L.) cultivées en Algérie. Agric. For. J. Vol. 3, No.1.pp. 15-22. E-ISSN 2602-5795.

Coulibaly K., Vall E., Autfray P., Sedogo P.M., 2012. Performance technico- économique des associations maïs/niébé et maïs/mucuna en situation réelle de culture au Burkina Faso : potentiels et contraintes, Tropicultura, 30 (3) : 147-154.

Danbe N., Yakouba O., Sobda G., Basga S.D., Lendzemo V., Kaouvon P., Dickmi Vailam C., Suh C., Djonnewa A., Youri A., Kaboui A. (2018). Caractérisation de la diversité phénotypique et génotypique du Sorgho pluvial dans la zone soudano-sahélienne du 
Caméroun. Journal of Applied Biosciences. $129: 12973-12981$. ISSN 1997-5902.

DGESS/MAAH, 2017. Base de données campagne agricole de référence 2015-2016. Février 2017.

FAOSTAT, 2017. http://www.faostat.fao.org.consulté le 10/06/2019.

Gate P., Cazabionca H., Deleen C., 1993. Interprétation des corrélations entre rendement et composition isotopique du carbone des grains. Diversité génétique et amélioration variétale, Montpellier (France). 15-17. Dec. 1992. Ed. INRA, Paris. 64:61-73.

Gueye T., Sine B., Cisse N., Diatta C., Ndiaye S., 2016. Characterization of Phenotypic Diversity of Sorghum Collection for Developing Breeding Material. Int. J. Sci.5, 38-48. https://doi.org/10.18483/ijSci.931.

Hani Ahmed Mohamed El Telib, 2000. Effect of time of nitrogen application on growth, yield and quality of forage sorghum cultivars. Master of Science (M. Sc.), University of Khartoum, pp. 66.

Hansen J.W., 2002. Realizing the potential benefits of climate prediction to agriculture: issues, approaches, challenges. Agric. Syst. 74, 309330.https://doi.org/10.1016/S0308-521X (02)00043-4.

IIFA, 2014.La fertilisation et les sols : les experts en parlent. International Fertilizer Industry Association, 28 rue Marbeuf, 75008, Paris, France. $8 \mathrm{p}$.

Innes T. M. T., Blakwell R. D., 1981. The effect of drought on the water use and yield of sprng wheat genotype. J. Agri. Sci. Camb. , 96: 603$610 . \quad$ http://www.minagri. $\mathrm{dz} / \mathrm{pdf} / \mathrm{BMI} / \mathrm{ITGC} /$ bulletin4_decembre 2013.pdf.

Kiébré Z, 2016. Etude De La Diversité Génétique d'une Collection De Caya Blanc (Cleome gynandra L.) du Burkina Faso. Thèse Unique de Doctorat, Université de Ouagadougou (Burkina Faso), 139p.

Kondombo, 2001. Evaluation agromorphologique et fourragère de 194 lignées recombinantes de sorgho. Mémoire d'Ingénieur du développement rural. Univ. Polytechnique de Bobo., 106p.

Koulibaly B., 2014.Situation de la fertilisation et de gestion de la fertilité des sols au Burkina Faso, INERA, Communication à la 7è réunion bilan du PR-PICA du 16 au 18 Avril 2014, Dakar. 29 $p$.

Ndiaye, 2018. Performances agronomiques et stabilité de génotypes de Sorgho (Sorghum bicolor (L.)) au Sénégal : une étude des interactions génotypes-environnement. Journal of. Appllied Biosciences. 125 : 12617-12629.

Nebié B., Gapili N., Traore R.E., Nanema K.R, BationoKando P., Sawadogo M. et Zongo J.D., 2012. Diversité phénotypique des sorghos à grains sucrés du centre nord du Burkina Faso. Sciences et techniques, sciences naturelles et agronomie vol $32, \mathrm{~N}^{\circ} 1$ et 2 .

Sawadogo N., Nebié B., Kiébré M., Bationo-Kando P., Nanema KR., Traoré RE., Gapili N., Sawadogo M. et Zongo JD., 2014a. Caractérisation agromorphologique des sorghos à grains sucrés (Sorghum bicolor (L.) Moench) du Burkina Faso. Int. J. Biol. Chem. Sci. 8 (5): 2183-219, 15p.

Sawadogo N., Nanema KR., Bationo P., Traore RE., Nebie B., Tiama D., Sawadogo M. et Zongo JD., 2014b. Évaluation de la diversité génétique des sorghos à grains sucrés [sorghum bicolor (L.) Moench] du Nord du Burkina Faso. Journal of Applied Biosciences, 84(1), 7654-7664.

Sawadogo N., 2015. Diversité génétique des sorghos à grains sucrés [sorghum bicolor (L.) Moench] du Burkina Faso. Thèse de Doct., Université de Ouagadougou, 194p.

Sawadogo N., Ouédraogo M.H., Traoré R.E., Nanéma K.R., Kiébré Z., Bationo-Kando P., Nebié B., Sawadogo M., Zongo J.D., 2017. Effect of Agromorphological Diversity and Botanical Race on Biochemical Composition in Sweet Grains Sorghum [Sorghum bicolor (L.) Moench] of Burkina Faso. Journal of BioScience and Biotechnology 6(1) : 263-269.

Sawadogo N, Batieno TJB, Kiébré Z., Ouédraogo M.H., Zida WPMSF., Nanéma KR,.Bationo-Kando P., Traore RE., Sawadogo M., Zongo JD.,2018. Assessment of genetic diversity of Burkina Faso sweet grain sorghum using microsatellite markers. African Journal of Biotechnology 17(12), 389-395.

Segda Z., Lompo F., Wopereis M. C. S. et Sedogo M. P ., 2001.Amélioration de la fertilité du sol par utilisation du compost en riziculture irriguée dans la vallée du Kou au Burkina Faso. Agronomie africaine, 13 (2) : 45-58. 
Slingo J.M., Challinor A.J., Hoskins B.J., Wheeler T.R., 2005. Introduction: food crops in a changing climate. Philos. Trans. R. Soc. 360, 19831989. https://doi.org/10.1098/rstb. 2005.1755.

Tiendrebeogo J., Sawadogo N., Kiebre M., Kabore B., Bationo/Kando P., Kiendrebeogo T., Ouedraogo M. H., Sawadogo M., 2018. Evaluation comparative de la production de grains et du fourrage de sorgho à grains sucrés du Burkina Faso. SPECIAL SIST 2017 SNA2_agrono27i 11: 36 pp. 261-271.

Vanlauwe B., Bationo A., Chianu J., Giller K.E., Merckx R., Mokwunye U., Ohiokpehai O., Pypers P., Tabo R., Shepherd K.D., Smaling E.M.A., Woomer P.L., Sanginga N., 2010. Integrated soil fertility management: Operational definition and consequences for implementation and dissemination. Outlook Agric. 39, 17-24. https://doi.org/10.5367/000000010791169998.

Zeinabou H., Mahamane S., Bismarck N.H., Bado B.V., Lompo F., Bationo A., 2014.Effet de la combinaison des fumures organo-minérales et de la rotation niébé-mil sur la nutrition azotée et les rendements du mil au sahel. International Journal of Biological Chemical Sciences, 8 (4): 1620-1632. 